
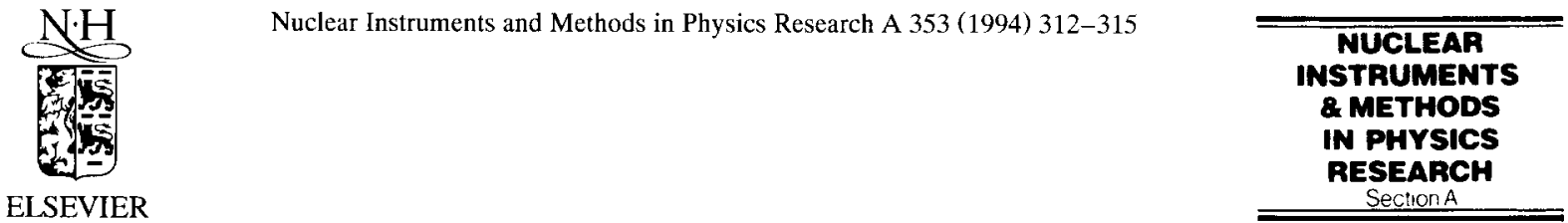

\title{
Microfocus X-ray sources for 3D microtomography
}

\author{
Michael J. Flynn a,b,*, Sean M. Hames ${ }^{\text {a,b }}$, David A. Reimann ${ }^{a}$, Scott J. Wilderman ${ }^{b}$ \\ ${ }^{d}$ X-ray Imaging Research Laboratory, Henry Ford Hospital, Detrott, MI, USA \\ ${ }^{\mathrm{b}}$ Department of Nuclear Engineering, University of Michigan, Ann Arbor, MI, USA
}

\begin{abstract}
An analytic model for the performance of cone beam microtomography is described. The maximum power of a microfocus X-ray source is assumed to be approximately proportional to the focal spot size. Radiation flux penetrating the specimen is predicted by a semi-empirical relation which is valid for $\mathrm{X}$-ray energies less than $20 \mathrm{keV}$. Good signal to noise ratio is predicted for bone specimens of 0.1 to $10 \mathrm{~mm}$ when scanned at the optimal energy. A flux of about $1 \times 10^{10}$ photons $/ \mathrm{mm}^{2} / \mathrm{s}$ is identified for $0.2 \mathrm{~mm}$ specimens. Cone beam volumetric microtomography is found to compare favorably with synchrotron based methods.
\end{abstract}

\section{Introduction}

Tomographic evaluation of biologic and geologic specimens, small industrial parts, and small animals can be accomplished with $\mathrm{X}$-rays. Three collaborating laboratories have developed methods for volumetric microscopic computed tomography ( $\mathrm{mCT}$ ) with a resolution down to about $12 \mu \mathrm{m}$ to study the three dimensional (3D) properties of bone material [1-3]. The method used employs a cone beam geometry with projections recorded in high magnification using an X-ray tube having a very small focal spot [3]. A similar approach has been used in England [4] and by investigators using modified electron microscopes [5].

In general, $\mathrm{X}$-ray mCT performance is limited by available X-ray flux. This has led to an interest in utilizing soft $\mathrm{X}$-ray synchrotron radiation. Using the Brookhaven synchrotron, Flannery has obtained resolutions approaching 1 $\mu \mathrm{m}$ [6]. Similar results have been achieved at Stanford and in Hamburg [7,8].

For single slice, narrow beam computed tomography (CT) done at optimal energy, Grodzins [9] has shown that the total number of $\mathrm{X}$-rays penetrating a specimen is constant if both the signal to noise ratio (SNR), $\mu / \sigma_{\mu}$, of the CT result and the ratio of the specimen diameter to the size of the elements in the CT image, $D / \delta$, are constant. In this paper, we analytically describe the $\mu / \sigma_{\mu}$, achieved with volumetric cone beam CT using electron impact $\mathrm{X}$-ray sources and different bone specimen sizes. The

\footnotetext{
* Corresponding author. E-mail mikef@rad.hfh.edu.
}

X-ray tube focal spot size, $f$, along with the target power is assumed to vary with specimen size. The optimal X-ray energy is identified for conditions in which the X-ray tube power is maintained as a constant, rather than assuming a constant source flux as was done by Grodzins. The radiation flux from microfocus X-ray sources is then compared to the flux available with synchrotrons.

\section{Methods}

Cone beam X-ray microtomograms are typically produced from a series of radiation transmission measurements obtained as a specimen is rotated through several hundred positions. The transmitted X-ray photons are detected by a two dimensional (2D) detector as an orthographic projection. This data is reconstructed using a cone beam algorithm [10] to produce a $3 \mathrm{D}$ estimate of the X-ray attenuation coefficients in the specimen.

In this section, we use a simplified model to estimate the SNR observed in projections and the propagation of this noise in CT reconstructions. In a well-designed system, measurement noise is dominated by statistical fluctuations associated with the number of radiation quanta detected in each detector element. The SNR for detectors recording a beam of radiation photons is then:

$S / \sigma_{s}=\left(A \phi t \epsilon_{\mathrm{d}}\right)^{1 / 2}$,

where $S$ is the signal in one detector element, $\sigma_{s}$ is the signal noise, $A$ is the area of a detector element, $\phi$ is the 
radiation flux in photons $/ \mathrm{cm}^{2} / \mathrm{s}, t$ is the exposure time in $s$, and $\epsilon_{\mathrm{d}}$ is the detector efficiency.

The radiation flux distribution incident to the detector may be written as:

$\phi(\theta, u, v)=\phi_{0}(\theta, u, v) \mathrm{e}^{-\int_{0}^{L} \mu(l, E) \mathrm{d} l}$,

where $l$ is the position within the object along a straight ray, in $\mathrm{cm}, \mu(l, E)$ is the linear attenuation coefficient in $\mathrm{cm}^{-1}$ at $l$ for energy $E, \phi_{0}$ is the flux produced by the $\mathrm{X}$-ray source in the absence of the imaged object in photons $/ \mathrm{cm}^{2} / \mathrm{s}, L$ is the thickness of the object, in $\mathrm{cm}$, and $(\theta, u, v)$ defines the path through the specimen at position $(u, v)$ in the detector and angle $\theta$.

The recorded signal in units of energy per detector element is simply $S=\left(A \phi t \epsilon_{\mathrm{d}}\right) E$, where $E$ is the energy per photon. It is common to define the projection as the line integral of the linear attenuation coefficient and estimate the projection values as a part of the data acquisition process:

$P(\theta, u, v)=\int_{0}^{L} \mu(l, E) \mathrm{d} l=\ln \left(\frac{S_{0}(\theta, u, v)}{S(\theta, u, v)}\right)$.

The noise for the projection measurement can thus be written as:

$\sigma_{p}=\sigma_{s} / S=1 /\left(A \phi t \epsilon_{\mathrm{d}}\right)^{1 / 2}$.

The convolution back-projection algorithm commonly used to reconstruct the value of the linear attenuation coefficient function within the object, $\mu(x, y)$, from a set of projections, $P(\theta, u, v)$, can be written in discrete form as:

$\mu(x, y) \cong \frac{\pi}{\mathrm{v}} \sum_{k=1}^{\mathrm{v}} P^{*}(\theta, u, v)$,

where $\mathrm{v}$ is the number of projection views, $P^{*}(\theta, u, v)$ is the projection at $(\theta, u, v)$ convolved with a reconstruction filter, and $(x, y)$ is the position within a specimen.

We use at this point equations applicable to $2 \mathrm{D}$ reconstruction from parallel beam projection measurements. However, if all positions are corrected for magnification to effective positions within the specimen, the results we obtain are equivalent to those obtained for cone beam solutions. In a manner similar to the derivation of Chesler [11], the noise in the reconstructed value for $\mu$ can be derived from $\mathrm{Eq}$. (5) as:

$\sigma_{\mu}=\frac{1}{2^{3 / 2} \delta v^{1 / 2}} \frac{\sigma_{s}}{S}=\frac{1}{2^{3 / 2} \delta v^{1 / 2}} \frac{1}{\left(A \phi t \epsilon_{\mathrm{d}}\right)^{1 / 2}}$,

where $\delta$ is the spacing of 3D data in the reconstruction array. This result is specific to the center of relatively uniform objects, where $\phi=\phi(\theta, 0,0)$ and assumes a $\sin \left(\omega_{\tau}\right) / \omega_{\tau}$ functional shape to the filter used in performing the convolution.
A relationship between electrical current and $\phi_{0}$ is needed so that power may be maintained as an independent variable in this analysis. For target elements below a $Z$ of about 50 , a large fraction of the emitted radiation is at the characteristic energy [12] and the bremsstrahlung radiation can be removed by thin metal filters. Using Michette's interpretation of an empirical relationship first reported by Green $[13,12]$ along with the classical relationship between characteristic energy and atomic number of $E_{\mathrm{c}} \propto(Z-1)^{2}$ $[14,15]$ and an approximation for atomic number of $A=2 Z$ we have derived the following approximate formulation:

$K_{\alpha}\left(E_{\mathrm{c}}\right)=9.23 \times 10^{-4} \frac{E_{\mathrm{c}}^{113}}{E_{\mathrm{c}}^{2}+72.1} f_{\mathrm{a}}$,

where $E_{\mathrm{c}}$ is the characteristic energy, $K_{\alpha}(\cdot)$ is the source emission strength in photons/electron/sr, and $f_{\mathrm{a}}$ is a correction for self absorption of radiation in the target. This equation assumes that the electron accelerating voltage to be employed is three times the characteristic energy. In this simple model we have assumed a constant self-absorption correction term of $f_{\mathrm{a}}=0.5$ for all targets.

The specimen has been modeled as a cylinder containing either regions of dense bone $\left(\rho_{\mathrm{b}}=2.18 \mathrm{~g} / \mathrm{cm}^{3}\right)$ or methacrylate $\left(\rho_{\mathrm{m}}=1.18 \mathrm{~g} / \mathrm{cm}^{3}\right)$. The attenuation coefficients used for each material were computed from the equation $\mu_{\mathrm{b}}=2.082 \times 10^{4} \rho_{\mathrm{b}} E_{\mathrm{c}}^{-2795}$ for bone and $\mu_{\mathrm{m}}=$ $2.695 \times 10^{3} \rho_{\mathrm{m}} E_{\mathrm{c}}^{-295}$ for methacrylate. These relationships were based on published values of the attenuation coefficients for the elements [16] in conjunction with our composition data on cortical bone and methacrylate resin. They are valid for energies below about $20 \mathrm{keV}$ and thus for X-ray targets with a $Z$ less than about 45 .

Bone specimen sizes ranging from 0.1 to $20 \mathrm{~mm}$ and bone volume $(\mathrm{BV})$ fractions ranging from 0.1 to 1.0 have been considered. The ratio $D / \delta$ has been assumed constant and equal to 256 . The total scan time, $v t$, has been fixed at $40 \mathrm{~min}$. The distance of the specimen from the focal spot, $d_{f}$, has been set such that $D / d_{f}=2 \tan 5^{\circ}$ (i.e. $d_{f}$ varied from 7 to $11 \mathrm{~mm}$ ) which assures that errors associated with cone beam reconstruction are minimal [17].

The focal spot size, $f$, needed for each specimen size, $D$, dictates the maximum $\mathrm{X}$-ray tube power. We assume that a high magnification geometry is employed with $f=\delta$ and thus $f$ varies from 0.4 to $78 \mu \mathrm{m}$. For small focal spot sizes, heat dissipation is predominantly radial for a stationary target and approximately proportional to $f$ [18]. Based on operational data from a Feinfocus model 160 in our laboratory, we have assumed that the maximum power in $\mathrm{W}$ is $1.4 f^{088}$ for $f$ in $\mu \mathrm{m}$. This is appropriate for a variety of target metals such as $\mathrm{Mo}, \mathrm{Y}, \mathrm{Ge}, \mathrm{Cu}, \mathrm{Cr}$, and $\mathrm{Ti}$ which have $\mathrm{K}_{\alpha}$ characteristic emissions at $17.5,15,10,8,5.4$, and $4.5 \mathrm{keV}$ respectively.

For this study, we have first determined the tube current in $\mathrm{mA}$ from the target power in $\mathrm{W}$. The $\mathrm{X}$-ray tube output was then computed from Eq. (7), the attenuation of 


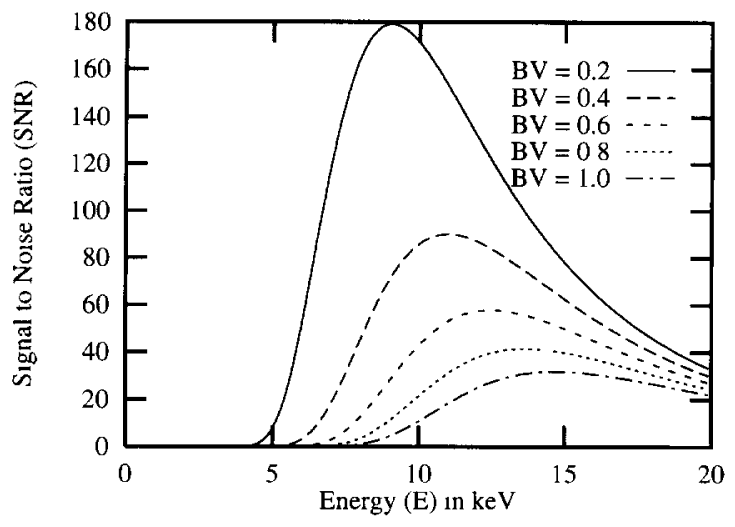

Fig. 1 . SNR vs. $E_{\mathrm{c}}$ for a $1 \mathrm{~mm}$ diameter specimen.

the X-ray beam by the specimen computed from Eq. (2), and the SNR computed by dividing $\mu_{\mathrm{b}}$ by the noise determined from Eq. (7).

The detector elements are assumed to be scaled down in size in proportion to the magnification of the object such that the detector element area, $A$, is equal to $\delta^{2}$. In effect, this scales the response of the detector so that it is at the center of rotation of the specimen and makes the detector element size equivalent to the spacing of points in the reconstruction. A constant detector efficiency of 0.5 was assumed. Eqs. (2) and (6) can be rewritten for convenience, using normalized variables, as:

$\left(\frac{\mu_{\mathrm{b}}}{\sigma_{\mu}}\right)^{2}=2^{3} \frac{\left(\mu_{\mathrm{b}} D\right)^{2}}{\mathrm{e}^{\int \mu \mathrm{d} l}}\left(\frac{\delta}{D}\right)^{4} \epsilon_{\mathrm{d}} N_{\mathrm{T}}$,

where $N_{\mathrm{T}}=\mathrm{v} t \phi_{0} D^{2}$ is the total number of X-rays passing into an object presenting an area of $D^{2}$ during a mCT scan.

\section{Results}

The sensitive effect of energy on the SNR, $\mu_{\mathrm{b}} / \sigma_{\mu}$, is illustrated in Fig. 1 for a $1 \mathrm{~mm}$ diameter object having

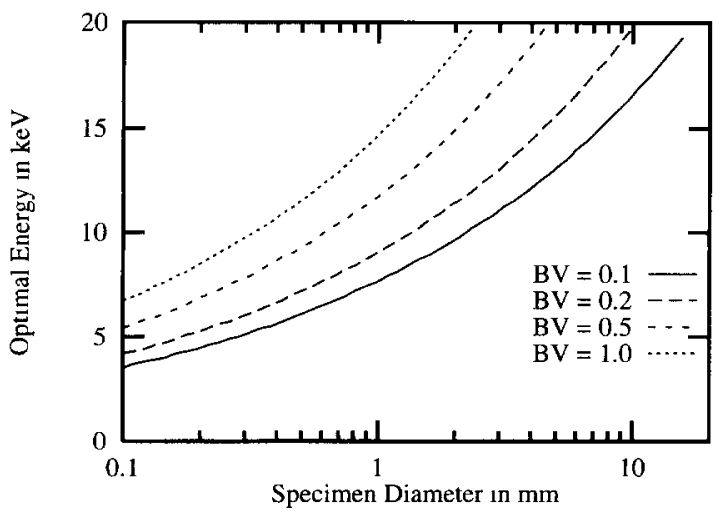

Fig 2. Energy at which the maximum SNR occurs vs. diameter.

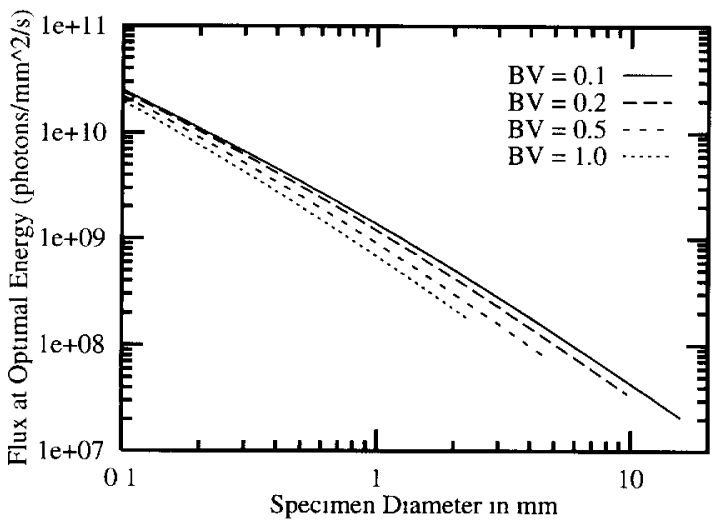

Fig. 3. Flux at the position of the specimen vs. diameter

different BV fractions. This result was obtained for a constant target power of $4.6 \mathrm{~W}$ and for $\delta$ and $f$ equal to $3.9 \mu \mathrm{m}$. Fig. 2 illustrates the energy value which produces maximal SNR (i.e. the optimal energy). The specimen transmission, $\mathrm{e}^{-\int \mu \mathrm{d} l}$ is between 0.08 and 0.14 at this energy.

The SNR value and the radiation flux at the center of the object in the absence of the object as a function of the specimen diameter for scans performed with the optimal energy are illustrated in Figs. 3 and 4. For these results, the power of the X-ray source varies with object diameter. The SNR at optimal energy is seen to increase with specimen diameter. This is due to the combined effects of higher source strength at higher optimal energy and higher flux at the increased power allowed with tubes having a larger focal spot size. The decrease in flux at the larger source to object distances used for larger objects is otherwise compensated for by the larger voxel size used with larger objects.

Our analysis predicts a flux at the specimen position of about $1 \times 10^{10}$ photons $/ \mathrm{mm}^{2} / \mathrm{s}$ for specimens of 0.1 to $0.3 \mathrm{~mm}$ diameter. In comparison, investigators doing $\mathrm{mCT}$ at synchrotron laboratories have reported using a flux in the range from 0.3 to $30 \times 10^{10}$ photons $/ \mathrm{mm}^{2} / \mathrm{s}[19,7,20]$.

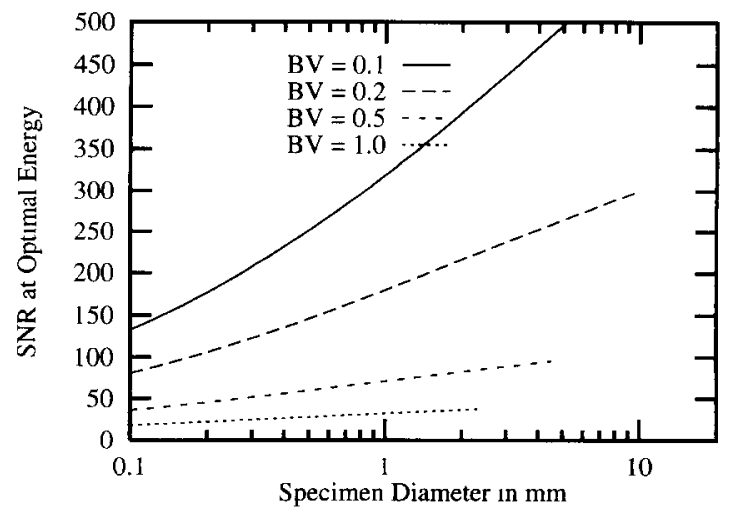

Fig. 4. SNR obtained with an optimum energy vs. diameter. 
Moreover, the SNR predicted for the method modeled is equal to or much greater than that needed for the evaluation of bone.

\section{Discussion}

The magnification geometry assumed in this study makes efficient use of the X-ray source. The alternative is a contact geometry with a large $f$ for which detail is observed with small detector elements. $f$ can then be increased in proportion to $d_{\mathrm{f}}$ however, the flux at the specimen decreases with $d_{\mathrm{f}}^{2}$ while the power, and thus the flux, increases only in proportion to $f^{n}$ where $n=1,3 / 2$, or 2 for a microfocus, rotating anode [21], or large stationary targets. As a result, the available flux through small specimens is better for a microfocus source and a large detector in a magnification geometry than for large source and a small detector in a contact geometry.

An important advantage of high magnification, cone beam geometry is the ability to use large detectors which can absorb a large fraction of the X-ray beam. With the cone beam geometry, a single detector with large, thick elements can be used to image specimens of various size. For example, a detector with element thickness and width of $50 \mu \mathrm{m}$ has an $\epsilon_{\mathrm{d}}$ of about 0.90 . Because of the parallel $\mathrm{X}$-ray beam emerging from synchrotron radiation sources, thin high resolution detectors have been necessary despite their poor efficiency [7]. A $1 \mu \mathrm{m}$ thick detector with a 1 $\mu \mathrm{m}$ resolution may be needed for small detail synchrotron mCT and such detectors will have an $\epsilon_{\mathrm{d}}$ of about 0.05 . Furthermore, a detector in a parallel beam system cannot be simply adjusted for the examination of specimens which have significantly different diameter.

Noting that small specimens require a higher incident radiation flux in order to obtain the same total amount of radiation penetrating the specimen, Grodzins concluded that only synchrotron sources would have sufficient flux to perform mCT on small objects [9]. Subsequently, many authors have reaffirmed the notion that synchrotron X-ray sources are greatly superior to $\mathrm{X}$-ray impact sources for mCT $[6-8,20]$. In general, we believe that these authors have not fully acknowledged the increase in flux that is achieved when small specimens are placed close to a microfocus source, the advantage of radial heat transfer in microfocus $\mathrm{X}$-ray sources relative to line-focus sources, and the value of using detectors with high efficiency which is made possible by high magnification in a cone beam geometry. We account for these factors in the analysis presented here and conclude that cone beam, volumetric $\mathrm{mCT}$ is a practical method for examining small specimens and compares favorably with synchrotron based methods.

\section{References}

[1] S.A. Goldstein, L.A. Feldkamp, A.M. Parfitt et al., J. Bone Mineral Res. 4 (1989) 3.

[2] J.L. Kuhn, S.A. Goldstein, L.A. Feldkamp et al., J. Orthop. Res. 8 (1990) 833.

[3] M.J. Flynn, D.A. Reimann, A.M. Parfitt et al., Proc 1992 IEEE Nucl. Sci. Symp. and Med. Imag. Conf., pp. 1292, 1993.

[4] E.J. Morton, S. Webb, J.E. Bateman et al., Phys. Med. Biol. 35 (1990) 805.

[5] P.C. Cheng, T.H. Lin, D.M. Shinozaki et al., Scanning 13 (1991) I-10.

[6] B.P. Flannery, H.W. Deckman, W.G. Roberge et al., Science 237 (1987) 1439.

[7] J.H. Kinney, Q.C. Johnson, M.C Nichols et al., Rev. Sci. Instr. 60 (1989) 2471.

[8] K. Engelke, M. Lohmann, W.R. Dix et al., Rev. Sci. Instr. 60 (1989) 2486.

[9] L. Grodzins, Nucl. Instr. and Meth. 206 (1983) 541.

[10] L.A. Feldkamp, L.C. Davis and J.W. Kress, J. Opt. Soc. Amer. A 1 (1984) 612.

[11] D.A. Chesler, S.J. Riederer and N.J. Pelc, J. Comput. Asst. Tomogr. 1 (1977) 64.

[12] M. Green and V.E. Cosslett, Proc. Phys. Soc. London 78 (1961) 1206.

[13] P.J. Duke and A.G. Michette (eds.), Modern Microscopies: Techniques and Applications (Plenum, New York, 1990).

[14] H.G. Moseley, Philos. Mag. 26 (1913) 1024.

[15] H.G. Moseley, Philos. Mag. 27 (1914) 703.

[16] W.H. McMaster, N.K. Del Grande, J.H. Mallett et al., Technical Report UCRL-50174, Lawrence Livermore Laboratory (1970)

[17] Hui Hu, PhD thesis, The University of Utah (1989).

[18] S. Whitaker, Medical Imaging II, SPIE Vol. 914 (1988) 565.

[19] K. Engelke and M. Lohmann, Nucl. Instr. and Meth. A (1989) 380.

[20] H.W. Deckman, J.H. Dunsmuir, K.L. D'Amico et al., Mater. Res. Soc. Symp. Proc., Vol. 217 (Materials Research Soc1ety, 1991) p. 97.

[21] M. Braun, Medical Imaging IV, SPIE Vol. 70 (1975) 65. 